\title{
Individualized robot-assisted training for MS- and stroke patients in I-TRAVLE
}

\author{
Sofie Notelaers* Tom De Weyer* Johanna Octavia* Karin Coninx ${ }^{*}$ \\ Hanne Bastiaens ${ }^{\ddagger}$ Peter Feys ${ }^{\ddagger}$ \\ (*)EDM, UHasselt-IBBT, Belgium \\ $(\ddagger) R E V A L, P H L$ university College \& BIOMED, UHasselt, Belgium \\ E-mail: \{sofie.notelaers, tom.deweyer, johanna.octavia,karin.coninx\}@uhasselt.be, \\ \{hanne.bastiaens,pfeys\}@mail.phl.be
}

\begin{abstract}
Persons with central nervous deficits, such as $M S$ and stroke patients, can benefit a lot from suitable training approaches that enhance their ability to perform activities in daily life. Personalized training, in accordance with the individual capabilities of the patient is a key issue in this context. We propose several techniques for individualization, including adaptive training games. Evaluations with patients and therapists reveal appreciation for the resulting Individualized, Technology-supported and RobotAssisted Virtual Learning Environments (I-TRAVLE system).
\end{abstract}

\section{Introduction}

Diseases and conditions resulting in individually very different symptoms, such as MS and stroke, raise the need for individualized training programs and training support. Multiple Sclerosis (MS) is a chronic and progressive disorder of the central nervous system, resulting in symptoms such as impairments of strength, muscle tone, sensation, co-ordination, balance, as well as visual and cognitive problems. A stroke is a sudden loss of brain function(s) due to disturbance in the blood supply to the brain. As a result, the affected area of the brain is unable to function, leading to inability to move one or more limbs on one side of the body, inability to understand or formulate speech, inability to see one side of the visual field, and cognitive impairments. An important part of therapy in both stroke and MS consists of physical and occupational training including exercise therapy. Training duration and training intensity are considered to be key factors for a successful neurological rehabilitation [1]. In this regard, a difficult to treat problem is upper limb dysfunction, which has immediate impact on functional independence in daily life.

In the context of the Interreg IV project "Rehabilitation robotics" we investigate how patientcentric robot-assisted rehabilitation can fulfill these needs and contribute to the motivation of the patient to keep up the training efforts. Because robot-assisted rehabilitation and virtual environment (VE) technologies have proven to be promising tools in addition to traditional therapy to create an even more effective exercise training [2][3], these technologies are combined in our I-TRAVLE system [4]. Using a haptic robot as the central hardware component, a software and hardware system setup has been realized to support systematic and personalized training for MS and stroke patients. The patient interface gives access to training exercises and games in virtual environments using haptic feedback [4]. A specific selection of individualized and motivating training exercises is necessary to strive for a successful rehabilitation trajectory. In our haptics-based rehabilitation system we provide basic training exercises which include only one skill component, but also more advanced training exercises (or games) which combine multiple skill components. The module for therapists allows us to define, to personalize, and to monitor the training [5].

As patients (and therapists) are the primary target users of I-TRAVLE, it is of utmost importance to serve their needs. Therefore, the development methodology being used adheres to user-centered design and involves patients as well as therapists, clinicians, software- and interaction specialists.

A detailed description of the different phases of the development process, including patient studies to assess the clinical relevance and usability of the interactive system, and of the details of the resulting system, is beyond the scope of this paper.

After a description of the overall system setup, we highlight in this paper the components of our approach

This is an Open Access article distributed under the terms of the Creative Commons Attribution-Noncommercial License 3.0, which permits unrestricted use, distribution, and reproduction in any noncommercial medium, provided the original work is properly cited. 
to individualize the patient's training on several levels, and we describe the corresponding features of the interactive software application. Personalization of the workspace, using difficulty levels and adaptivity in the training software are key issues of the approach.

\section{System Setup}

For the purpose of understandability, we will first present a general overview of the system setup and the training exercises, and then focus on individualization.

Figure 1 shows an overview of the system setup. The central component of the system is the MOOG HapticMaster [6] that functions as an output device, providing haptic feedback during the training by guiding or hindering the patient with exerted forces, but also as an input device, allowing the patient to interact with the software applications that deliver the training exercises. The HapticMaster was mainly chosen because of its abilities to provide a relatively large workspace and to exert a desired range of forces.

The HapticMaster is equipped with an ADL gimbal, which is the peripheral device where the user can place her hand and that is designed by MOOG to support activities of daily life (ADL). The patient is sitting in front of the HapticMaster in such a way that the bar of the device cannot come in contact with the patient. The patient's hand is placed in the gimbal and secured into the attached brace.

A large display / TV screen is placed behind the HapticMaster, approximately $1.5 \mathrm{~m}$ in front of the patient. This Full HD 40" TV screen (Samsung) is used to project the exercises to the patients. The smaller screen that is visible in Figure 1 to the left of the large patient's display is the therapist interface, which can also be located in another place but usually it will be nearby the location where the patient is training under supervision of the therapist.

Figure 1 also shows the use of a Sling device manufactured by FOCAL [7] for those patients needing a substantial anti-gravity support to be able to perform the training exercises.

\section{Workspace}

Patients' abilities for upper limb usage differ a lot for individual patients, and some will have, a smaller range of motion than others. So (1) a way to measure these differences needs to be determined, and (2) afterwards training expectations and the setup of the training program has to be adjusted according to these measurements. This personalization approach ensures that the effort for training exercises is within the capability of the patient, and does not ask for movements of the arm that are impossible or harmful.

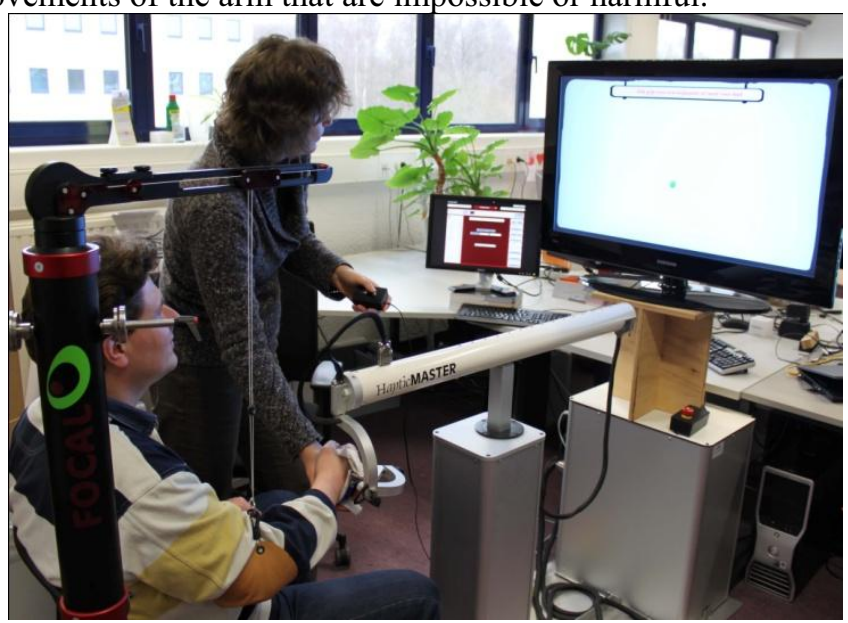

Figure 1: Hardware Setup, including haptic master, sling and visual display

To realize the first aspect of the personalization infrastructure, measuring the individual differences between patients, we created calibration applications to measure the active range of motion (ROM) of the patient. These applications will be used by the therapist before the patient executes the training exercises.

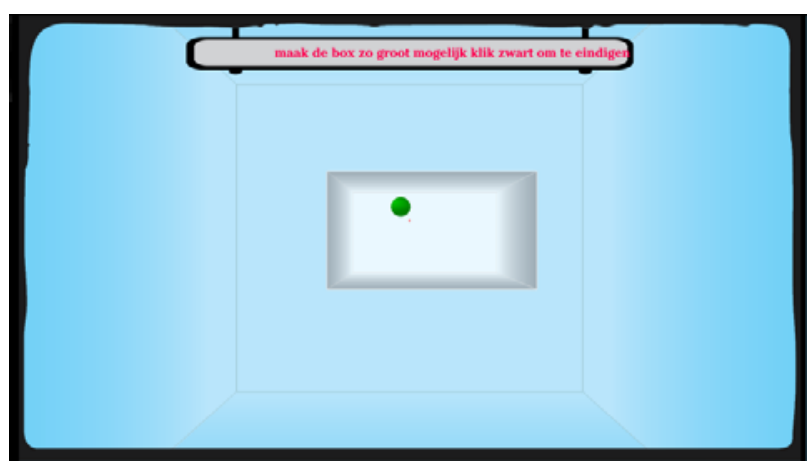

Figure 2: Active ROM Application

To determine the active ROM (Figure 2) the patient will have to move his arm as far as possible in every direction, without using compensations of the upper body. The HapticMaster device follows the movement but does not deliver extra support for the patient. The software records the outer limits and stores this information in a central database. Each subsequent training exercise will take these values into account, ensuring the distance of movements within the exercises does not surpass these values. This is done by scaling the actual movements of the HapticMaster to the required movements whilst the patient performs the exercise. 


\section{Difficulty levels}

Once the individual workspace is determined and the training program is set by the therapist the patient can start training. To set up a training program the therapist can choose among several exercises provided by the system (Error! Reference source not found.). Selected exercises take into account the desire of the patient to train certain activities of daily life. Each exercise trusts on certain parameters that can change its difficulty level. Providing customizable parameters thus ensures personalization to better suit the patients' desires and capabilities.

Though introducing flexibility, setting the extensive number of parameters each time an exercise starts may become a tedious task. Therefore three categories of parameters are defined (general, haptic and training volume parameters), where each category has 5 levels of predetermined values as illustrated in the central part of Figure 3. Classifying parameters in this way supports flexible creation of diverse training programs. General parameters include settings about the visual representation and auditory feedback, for example show the optimal path that the user needs to follow. Haptic parameters include the weight of an object in the virtual environment or supporting the movements of the patient along the optimal path. Finally the training volume parameters can include length of the exercise or the number of repetitions of a certain movement. A detailed overview of these parameters can be found in [5].

The therapist takes the initiative to select the appropriate levels for each exercise while creating a training program for a particular patient. On choosing a difficulty level the system will automatically set the corresponding parameters. To facilitate an even more advanced level of personalization each of the parameters within a difficulty level can be adapted by the therapist. These setting are then stored under the specific individual patient profile for future use.

\section{Adaptivity in Penguin Painting}

In the above introduced personalization techniques, the therapist manually adjusts the difficulty levels to result in suitable, challenging and individualized rehabilitation programs. However, this dependency on the therapist's involvement can be minimized while tailoring of the game challenge can still be provided at the right time.

Based on dynamically measured and monitored performance of the patient, automatic adaptation of the difficulty level can be part of the optimization strategy for individualized training. To deliver proof of concept

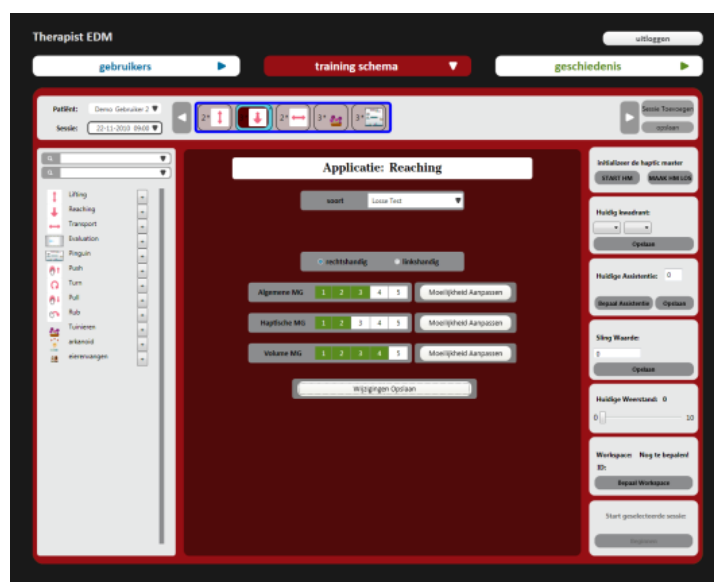

Figure 3: Therapist interface where a training program can be created

of this approach, this kind of adaptivity has been implemented in one of our training games namely the penguin painting exercise. After explaining the goal of the game, we will highlight the automatic adaptation technique that is demonstrated.

\subsection{Penguin Painting Exercise}

The goal of the exercise shown in figure 4 is to color the penguins correctly and collect as many points as possible within a certain time limit. The black penguins placed on the left side of the display have to be colored according to their belly by exercising appropriate skill components. The game includes small and big penguins where the former is much lighter to transport then the latter.

The user has to select a penguin on the shelf and dip it into the correct buckets located at the top and at the bottom of the game space. The top buckets will paint the upper part of the penguins while the bottom buckets will change the color of the lower part. Small buckets require a much more precise movement than their larger counterpart. The color of the penguin is only applied when holding it long enough into the bucket (to train stabilization of the upper limb). To provide some extra challenges in the game, a devil that captures the penguin might appear. Furthermore an eventual obstacle needs to be avoided to reach the buckets.

When the playing patient has completely colored the penguin, the character must be dropped on the correct platform to the right of the game space.

\subsection{Automatic adaptation}

To achieve a suitable challenge during the game we investigated the option of dynamically adjusting the difficulty of the game. Concretely, in our proof of 


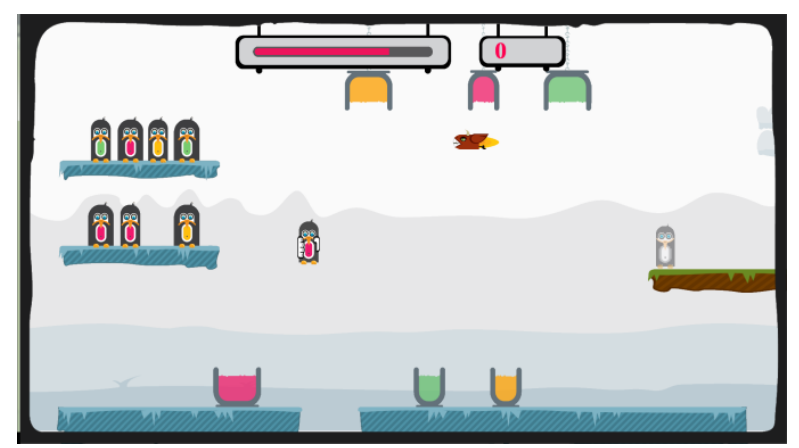

Figure 5: Penguin Painting Level 4

concept realization, this was done using automatic difficulty adjustments according to the players' actual performance and progress in the game. Examples of the difficulty adjustments are changing the ratio of small and big penguins, frequency of the devil, width of the buckets etc.

Seven levels of difficulty were defined ranging from very easy to very difficult. This technique brings along many opportunities to adapt for every individual patient, making it easier for weak patients or more difficult for stronger patients. Examples of predefined levels can be seen in Figure 4 and Figure 5.

Patients start their training on a certain level, and the system detects triggers for adjusting the level. The (currently basic) detection algorithm compares patient performance over the last two game trials or exercises. When there is no significant difference in performance the algorithm assumes that the patient is training on an appropriate level, otherwise it will increase or decrease the level accordingly by one level at a time. The performance is measured by the score, the average time to color a penguin, the average distance traveled, the amount of errors that are made, etc.

An informal test revealed promising results as patients as well as therapists appreciate the automatic level adjustments. Patients liked the diversity of levels and report enhanced motivation to continue training with the game. Further investigation is needed to optimize the adaptation algorithm and realize other kinds of adaptive games.

\section{Conclusions and Future Work}

We have described how different levels of individualization, ranging from parameters adjustable by therapists to automatically adapting game levels, have been integrated and successfully explored in our I-TRAVLE rehabilitation environment. Adaptation is promising, and inspires further research regarding adaptation triggers (where we started exploring EMG based fatigue prediction/detection) and adaptation algorithms.

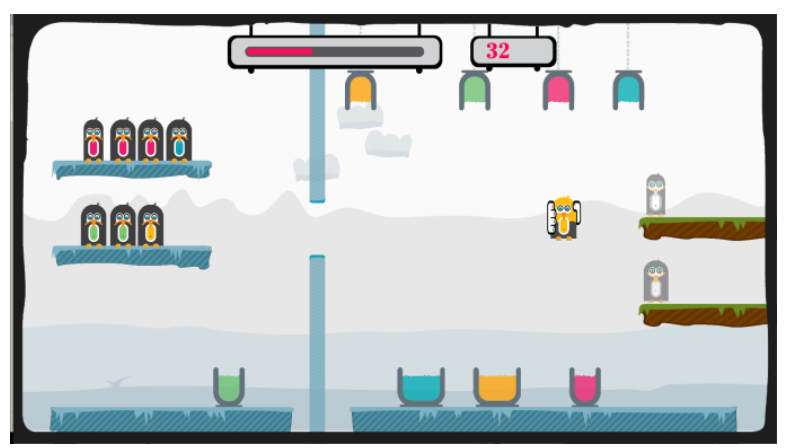

Figure 4: Penguin Painting Level 7

\section{Acknowledgments}

$<$ to be added in final manuscript $>$

\section{References}

[1] G. Kwakkel, R. van Peppen, R. Wagenaar, S. Dauphinee, C. Richards, A. Ashburn, K. Miller, N. Lincoln, C. Partridge, I. Wellwood, and P. P. Langhorne, "Effects of Augmented Exercise Therapy Time After Stroke: A Meta-Analysis", Stroke 35: 2529 2539; 2004

[2] J.H. Burridge and AM. Hughes, "Potential for new technologies in clinical praktisch." Current Opinion in Neurology, vol. 23, no. 62010.

[3] G. Kwakkel, B. J. Kollen, H. I. Krebs, Effects of robotassisted therapy on upper limb recovery after stroke: a systematic review. Neurorehabilitation and neural repair, Vol. 22, No. 2. (r 2008), pp. 111-121.

[4] P. Feys, G. Alders, D. Gijbels, J. De Boeck, T. De Weyer, K. Coninx, C. Raymaekers, V. Truyens, P. Groenen, K. Meijer, H. Savelberg, B. Op 't Eijnde, "Arm training in Multiple Sclerosis using Phantom: clinical relevance of robotic outcome measures ", Proc. of ICORR 2009, pp. 576 - 581, IEEE, ISBN 9781-42443789-4, Jun. 2009

[5] S. Notelaers, T. De Weyer, C. Raymaekers, K. Coninx, H. Bastiaens, \& I. Lamers (2010). Data Management for Multimodal Rehabilitation Games," Database and Expert Systems Applications, International Workshop on, pp. 137-141, 2010 Workshops on Database and Expert Systems Applications.

[6] P. Lammertse, E. Frederiksen, and B. Ruiter, "The hapticmaster, a new high-performance haptic interface," in Proceedings of Eurohaptics 2002, Edinburgh, UK, 2002.

[7] http://www.focalmeditech.nl/index.php/home/home/39sling

[8] T. De Weyer, S. Notelaers, K. Coninx, P. Feys, I. Lamers, G. Alders, R. Geers "Watering the flowers: virtual haptic environments for training of forearm rotation in persons with central nervous deficits", PETRA 2011, ACM, ISBN 978-1-4503-0772-7, May 2011. 